\title{
Nonparaxial spatial solitons and propagation-invariant pattern solutions in optical Kerr media
}

\author{
Bruno Crosignani* and Amnon Yariv \\ California Institute of Technology 128-95, Pasadena, California 91125 \\ Shayan Mookherjea \\ University of California, San Diego, Mail Code 0407, La Jolla, California 92093-0407
}

Received January 20, 2004

\begin{abstract}
We investigate nonlinear propagation in the presence of the optical Kerr effect by relying on a rigorous generalization of the standard parabolic equation that includes nonparaxial and vectorial terms. We show that, in the $(1+1)$-D case, both soliton and propagation-invariant pattern solutions exist (while the standard hyperbolic-secant function is not a solution). (C) 2004 Optical Society of America

OCIS codes: $190.0190,190.3270,190.5530$.
\end{abstract}

Monochromatic optical propagation in the presence of a refractive-index distribution $n(\mathbf{r})=n_{0}+\delta n(\mathbf{r})$ is usually described by the scalar parabolic equation. This is a by-product of the Helmholtz equation

$$
\nabla^{2} \mathbf{E}-\nabla \nabla \cdot \mathbf{E}+\left(\omega^{2} / c^{2}\right) n^{2}(\mathbf{r}) \mathbf{E}=0,
$$

and the scalar parabolic equation is derived from Eq. (1) in the paraxial approximation. More precisely, after writing the electric field as $\mathbf{E}(\mathbf{r}, t)=$ $\mathbf{A}\left(\mathbf{r}_{\perp}, z\right) \exp (i k z-i \omega t)$, where $\omega$ is the angular frequency, $k=(\omega / c) n_{0}$, and introducing $d$ as a typical length scale of variations in $n(\mathbf{r})$, we assume that the parameter $\eta=(\Delta n / n)(\lambda / d) \ll 1$, which expresses the smallness of the relative variation of the refractive index over a wavelength scale. Besides, if the field is initially (approximately) transversely polarized, we neglect its longitudinal component $A_{z}$ with respect to the transverse one $\mathbf{A}_{\perp}\left(\mathbf{r}_{\perp}, z\right)$, and we can derive, using the slowly varying approximation, the standard scalar parabolic equation:

$$
i \frac{\partial}{\partial z} \mathbf{A}_{\perp}+\frac{1}{2 k} \nabla_{\perp}^{2} \mathbf{A}_{\perp}+\frac{k}{n_{0}} \delta n(\mathbf{r}) \mathbf{A}_{\perp}=0 .
$$

We can then automatically satisfy the divergence equation $\nabla \cdot(\epsilon \mathbf{E})=0$ (where $\epsilon=\epsilon_{0} n^{2}$ ) by using it to derive the (small) longitudinal component $A_{z}$. If the smallness parameter $\eta$ becomes comparable to 1 , then the approach presented above, which represents only the lowest-order approximation in $\eta$, fails, and higher-order terms have to be added to account for nonparaxial contributions. The recent progress in nanotechnology and the possibility of fabricating optical structures with subwavelength features are compelling reasons for generalizing Eq. (2) to the nonparaxial regime. This can be done rigorously if, after splitting the field into a transverse and a longitudinal part, one is able to derive an equation for the transverse part alone to first order in $\partial / \partial z$ that contains terms to all orders in $\eta$. This task was recently carried out in the general case (linear or nonlinear) of a tensorial refractive-index distribution, $\overleftrightarrow{\mathbf{n}}(\mathbf{r})$, in a manner that fully preserved the vectorial nature of the problem. ${ }^{1}$ The resulting equation coincides with a vectorial equation derived by different authors ${ }^{2}$ for an isotropic refractive-index distribution, i.e., for a scalar $n(\mathbf{r})$ and up to the second order in $\eta$.

The general equation worked out in Ref. 1 can be used to study nonlinear optical propagation in the presence of the optical Kerr effect. In particular, although it is well established that the associated parabolic paraxial equation, also known as the nonlinear Schrödinger equation, admits of spatial soliton solutions of the hyperbolic-secant kind in the $(1+1)$-D case, it remains to be seen whether this is still the case if nonparaxial effects are taken into account. There have been many recent attempts to derive equations specifically aimed at describing propagation in the nonlinear Kerr regime beyond the standard paraxial scalar approximation, resulting in the existence of a number of different equations that do not agree with one another. The equation that Ciattoni et al. derived in Ref. 3 agrees with the one recently derived by means of an independent approach. ${ }^{4}$

To analyze the $(1+1)$-D case we assume the propagating field to be initially polarized along the $x$ direction and the $y$ component to remain negligible over the distance of propagation (which is the case for at least a few diffraction lengths; see Ref. 3). Then, the $x$ component $A_{x} \equiv A$ obeys the equation ${ }^{3}$

$$
\begin{aligned}
& \left(i \frac{\partial}{\partial z}+\frac{1}{2 k} \frac{\partial^{2}}{\partial x^{2}}\right) A(x, z)=-k \frac{n_{2}}{n_{0}}\left(|A|^{2} A+\frac{1}{3 k^{2}}\right. \\
& \left.\times|A|^{2} \frac{\partial^{2}}{\partial x^{2}} A+\frac{8}{3 k^{2}} A\left|\frac{\partial}{\partial x} A\right|^{2}+\frac{5}{6 k^{2}} A^{2} \frac{\partial^{2}}{\partial x^{2}} A^{*}\right),
\end{aligned}
$$

where $n_{2}$ is the nonlinear refractive-index coefficient. We introduce the dimensionless coordinates and amplitude $\xi=k x, \zeta=k z$, and $U=\left(n_{2} / n_{0}\right)^{1 / 2} A$ and label with a prime partial differentiation with respect 
to $\xi$. Then, Eq. (3) can be recast in the form

$$
\begin{aligned}
i(\partial / \partial \zeta) U+(1 / 2) U^{\prime \prime} & =-|U|^{2} U-(1 / 3)|U|^{2} U^{\prime \prime} \\
& -(5 / 6) U^{2} U^{* \prime \prime}-(8 / 3) U\left|U^{\prime}\right|^{2},
\end{aligned}
$$

which coincides with the one derived in Ref. 4 . We look for solutions of the form $U(\xi, \zeta)=u(\xi) \exp (i \beta \zeta)$, where $u$ is a real function of $\xi$ and $\beta$ is a real parameter. Equation (4) simplifies to

$$
\begin{aligned}
-\beta u+(1 / 2) u^{\prime \prime} & =-u^{3}-(7 / 6) u^{2} u^{\prime \prime}-(8 / 3) u u^{\prime 2}, \\
\frac{\mathrm{d} f}{\mathrm{~d} u}+\frac{32}{3+7 u^{2}} u f & =-12 \frac{u\left(-\beta+u^{2}\right)}{3+7 u^{2}} .
\end{aligned}
$$

After introducing the new variable $f=(\mathrm{d} u / \mathrm{d} \xi)^{2}$, we can rewrite Eq. (5) as

$$
\begin{aligned}
f(u)= & -\frac{12}{\left(3+7 u^{2}\right)^{16 / 7}} \\
& \times \int_{u_{0}}^{u} \tilde{u}\left(-\beta+\tilde{u}^{2}\right)\left(3+7 \tilde{u}^{2}\right)^{9 / 7} \mathrm{~d} u \\
& +\frac{\left(3+7 u_{0}^{2}\right)^{16 / 7}}{\left(3+7 u^{2}\right)^{16 / 7}} f\left(u_{0}\right),
\end{aligned}
$$

which can be integrated. Since we are looking for bright soliton solutions (peaked envelopes), we can take $u_{0}$ to refer to the maximum value of the field envelope, where $f=(\mathrm{d} u / \mathrm{d} \xi)^{2}=0$. Then, Eq. (7) reduces to

$$
\begin{aligned}
f(u)= & -\frac{6}{\left(3+7 u^{2}\right)^{16 / 7}} \int_{u_{0}{ }^{2}}^{u^{2}}(-\beta+w)(3+7 w)^{9 / 7} \mathrm{~d} w \\
= & -\frac{6}{\left(3+7 u^{2}\right)^{16 / 7}}\left\{-\beta\left[G\left(u^{2}\right)-G\left(u_{0}^{2}\right)\right]\right. \\
& +u^{2} G\left(u^{2}\right)-u_{0}^{2} G\left(u_{0}^{2}\right) \\
& \left.+G\left(u_{0}^{2}\right) \frac{3+7 u_{0}^{2}}{23}-G\left(u^{2}\right) \frac{3+7 u^{2}}{23}\right\}
\end{aligned}
$$

where $G(x)=(3+7 x)^{16 / 7} / 16$. To find $\beta$ in terms of $u_{0}$ we impose the further condition that when $\xi \rightarrow \pm \infty$ both $u$ and its derivative (and thus $f$ ) go to zero. Thus, we obtain

$$
\begin{aligned}
\beta & =\frac{\int_{0}^{u_{0}{ }^{2}} w(3+7 w)^{9 / 7} \mathrm{~d} w}{\int_{0}^{u_{0}{ }^{2}}(3+7 w)^{9 / 7} \mathrm{~d} w} \\
& =\frac{16}{23} \frac{u_{0}^{2} G\left(u_{0}^{2}\right)}{G\left(u_{0}^{2}\right)-G(0)}-\frac{3}{23} .
\end{aligned}
$$

As expected, $\beta$ is always positive, and for both $u_{0} \gg 1$ and $u_{0} \ll 1$, one has $\beta=-(16 / 23) u_{0}^{2} \cong-(2 / 3) u_{0}^{2}$.
After inserting Eq. (9) into Eq. (8), we check that $f(u)$, defined above as $(\mathrm{d} u / \mathrm{d} \xi)^{2}$, is always positive as shown in Fig. 1, so that there exist soliton solutions. The envelopes can be numerically evaluated by integrating Eq. (8) and are shown in Fig. 2 for different values of $u_{0}{ }^{2}$. For large $u_{0}$, the width of the soliton is seen to exhibit a very slow dependence on $u_{0}^{2}$, unlike paraxial nonlinear Schrödinger equation solitons, where the width scales linearly as $1 / u_{0}$. To explain this result we observe (as shown in Fig. 3) that, for large $u_{0}$, we can approximately write $f(u)=\left(\alpha-c u^{2}\right)$, where, empirically, $c=1 / 4$, independent of $u_{0}$. This relationship holds over most of the range of $0<u<u_{0}$, except for small $u$ (i.e., the tail), and only $\alpha$ depends on the value of $u_{0}$. Then, this differential equation yields an explicit solution for the large- $u$ portion of the envelope, $u(\xi)=\sqrt{ }(\alpha / c) \cos (\sqrt{ } c \xi)$ with $\alpha=u_{0}^{2} / 4$, whose width is indeed independent of $u_{0}$. Conversely, by expanding the right-hand side of Eq. (8) for small values of $u_{0}$, one has $f(u)=(14 / 23) u_{0}^{2}\left(1-u^{2} / u_{0}^{2}\right)$, which can be integrated to approximately yield

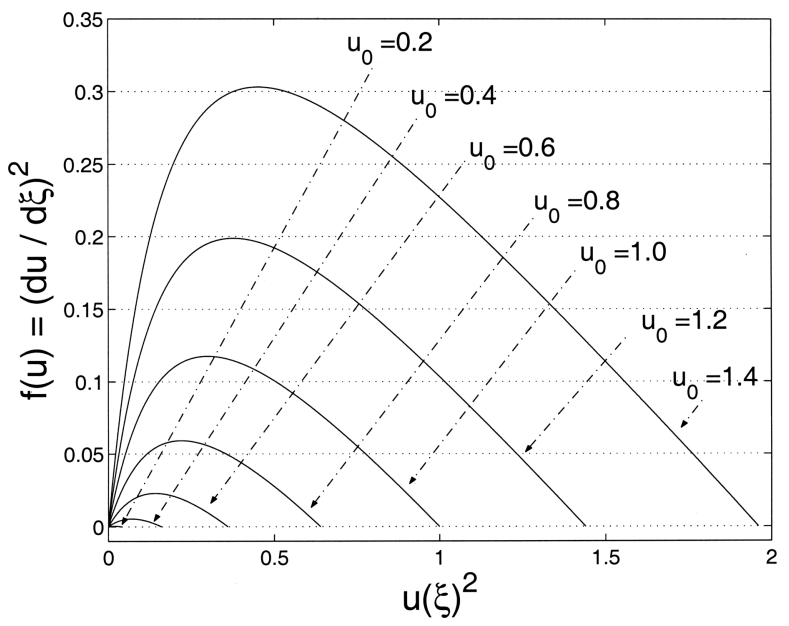

Fig. 1. $f(u) \equiv(\mathrm{d} u / \mathrm{d} \xi)^{2}$ plotted as a function of $u(\xi)^{2}$ for different values of $u_{0}$, the peak amplitude. The range of $u$ is from 0 to $u_{0}$ in each case, and $f(u)$ is always positive.

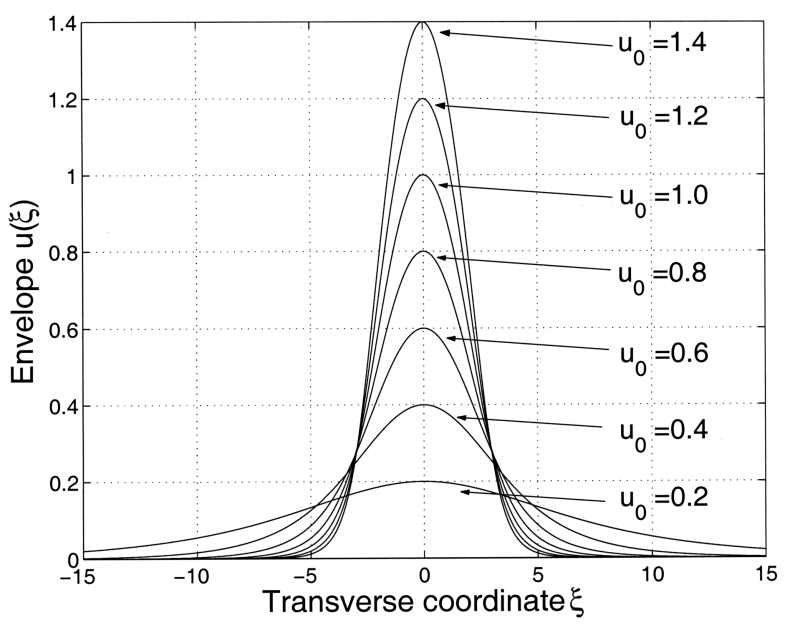

Fig. 2. Soliton envelope $u(\xi)$ obtained by solving $(\mathrm{d} u / \mathrm{d} \xi)^{2}=f(u)$, where $f(u)$ is obtained from Fig. 1. As the peak amplitude $u_{0}$ becomes comparable to 1 , the envelope widths approach a constant value. 


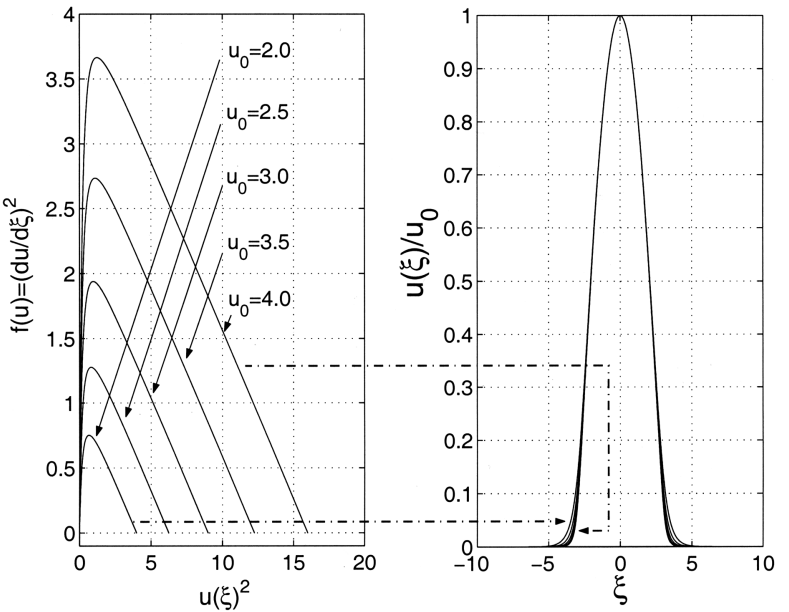

Fig. 3. For large values of $u_{0} \gg 1$ the normalized envelopes $u(\xi) / u_{0}$ have a constant width, independent of $u_{0}$.

$u=u_{0} \cos \left[(14 / 23)^{1 / 2} u_{0} \xi\right]$, so that the soliton width scales, as expected, with $1 / u_{0}$.

We also found that Eq. (4) admits of exact analytic solutions in the form of invariant-pattern beams, $U(\xi, \zeta)=\exp (i \beta \zeta) u_{0} \sin (\alpha \xi+\gamma)$, for any value of $u_{0}$ and provided that

$$
\alpha^{2}=\frac{6}{23} \cong \frac{1}{4}, \quad \beta=\frac{\alpha^{2}}{2}\left(\frac{16}{3} u_{0}^{2}-1\right) .
$$

Note that the numerical values given in Eqs. (10) coincide for large values of $u_{0}$ with those found for the spatial solitons. Since $u(\xi)$ does not go to zero as $\xi \rightarrow \pm \infty$, this solution describes a solitary wave, rather than a soliton, and it represents the nonlinear Kerr counterpart of the propagation-invariant pattern solutions of the exact scalar Helmholtz equation in a linear homogeneous medium (the one-dimensional analog of the two-dimensional diffraction-free Bessel beam). ${ }^{5}$ In dimensional notation, our invariantpattern solution reads as

$$
E(x, z)=\sqrt{n_{0} / n_{2}} u_{0} \exp [i(1-\beta) k z] \sin (\alpha k x+\gamma),
$$

which can be excited by the incidence from air of two plane waves intersecting at a specific angle $+\theta_{0}$ and $-\theta_{0}$ with respect to the $z$ axis, $E(x, z)=E_{0} \exp \left[i\left(k_{z} z \pm\right.\right.$ $\left.k_{x} x\right)$. By matching the tangential field components at the interface between air and the nonlinear medium, we conclude that $\tan \theta_{0}=\alpha n_{0} /\left(1-\alpha^{2} n_{0}^{2}\right)^{1 / 2}$, which limits the excitation from air to dielectric material with $n_{0} \leq 1 / \alpha \cong 2$. Angle $\theta_{0}$ is approximately $\pi / 4$ for $n_{0}=1.5$.

Various other equations similar to Eq. (4) but differing in the coefficients and in the presence of an extra nonlinear term of the kind $U^{\prime 2} U^{*}$ have been reported. ${ }^{6-10}$ In these cases as well, our analysis can be used to investigate soliton and invariant-pattern solutions. All these equations were derived by adopting a vectorial approach, without neglecting the polarization-mixing term in Eq. (1). Recently, it was claimed that $(1+1)$-D nonparaxial solitons of the standard hyperbolic-secant form exist, ${ }^{11}$ based on solving the nonlinear Helmholtz equation

$$
\left(\frac{\partial^{2}}{\partial z^{2}}+\frac{\partial^{2}}{\partial x^{2}}+k^{2}\right) E+2\left(n / n_{0}\right) k^{2} E=0,
$$

which is assumed to describe nonparaxial propagation through the presence of the second $z$-derivative term, usually neglected in the slowly varying approximation. This is true if the analysis is restricted to the scalar approximation, ${ }^{12}$ but actually both the tensorial nature of the nonlinear interaction and the presence of the polarization-mixing term, present in Eq. (1) but neglected in Ref. 11, play an important role in the nonparaxial regime ${ }^{3}$ and cannot be omitted.

In conclusion, by relying on a rigorous equation describing nonparaxial vectorial propagation in the presence of the Kerr nonlinearity, we have been able to show the existence of $(1+1)$-D spatial solitons and of invariant-pattern solutions in the nonparaxial regime. The spatial solitons that we have found differ remarkably from their paraxial counterpart in that their excitation requires, as long as $u_{0} \geq 1$, a width of the order of $\lambda / 2$, practically independent from their peak amplitude.

The authors are grateful to Alessandro Ciattoni and Eugenio DelRe for useful discussions. The work at the California Institute of Technology was supported by the U.S. Office of Naval Research. This project was partially supported by the Italian Ministry for Research through the Fondo per gli Investimenti della Ricerca di Base initiative and by the Italian Istituto Nazionale di Fisica della Materia through the SEH ("Solitons embedded in holograms") project.

*B. Crosignani (bcross@caltech.edu) is also with the Dipartimento di Fisica, Universita dell'Aquila, 67100 Aquila, Italy, and the Istituto Nazionale di Fisica della Materia, Unita di Roma "La Sapienza," 00185 Rome, Italy.

\section{References}

1. A. Ciattoni, P. Di Porto, B. Crosignani, and A. Yariv, J. Opt. Soc. Am. B 17, 809 (2000).

2. A. Yu. Savchenko and B. Ya. Zel'dovich, Phys. Rev. E 50, 2389 (1994).

3. A. Ciattoni, C. Conti, E. DelRe, P. Di Porto, B. Crosignani, and A. Yariv, Opt. Lett. 27, 734 (2002).

4. R. de la Fuente, R. Varela, and H. Michinel, Opt. Commun. 173, 403 (2000).

5. J. A. Stratton, Electromagnetic Fields (McGraw-Hill, New York, 1941).

6. S. Chi and Q. Guo, Opt. Lett. 20, 1598 (1995).

7. B. Crosignani, P. Di Porto, and A. Yariv, Opt. Lett. 22, 778 (1997).

8. S. Blair, Chaos 10, 570 (2000).

9. G. Fibich and B. Ilan, Physica D 157, 112 (2001).

10. K. Marinov, D. I. Pushkarov, and A. Shivarova, in Soliton-Driven Photonics, A. D. Boardman and A. P. Sukhorukov, eds. (Kluwer, Dordrecht, The Netherlands, 2001), pp. 293-316.

11. P. Chamorro-Posada, G. S. McDonald, and G. H. C. New, Opt. Commun. 192, 1 (2001).

12. T. A. Laine and A. T. Friberg, J. Opt. Soc. Am. B 17, 751 (2000). 